\title{
A Review of Techniques to Mitigate Jamming in Electromechanical Actuators for Safety Critical Applications
}

\author{
Yameen M. Hussain ${ }^{1}$, Stephen Burrow ${ }^{2}$, Leigh Henson ${ }^{3}$, Patrick Keogh ${ }^{4}$ \\ ${ }^{1,2}$ University of Bristol, Bristol, BS8 1TR, United Kingdom \\ yameen.hussain@bristol.ac.uk \\ Stephen.Burrow@bristol.ac.uk \\ ${ }^{3}$ Stirling Dynamics Ltd, Bristol, BS8 4HG, United Kingdom \\ Leigh.Henson@stirling-dynamics.com \\ ${ }^{4}$ University of Bath, Bath, BA2 7AY, United Kingdom \\ p.s.keogh@bath.ac.uk
}

\begin{abstract}
This paper presents a review of techniques to mitigate jamming in Electromechanical Actuators (EMA) for safety critical applications in aerospace. Published progress to date is evaluated, with the remaining challenges highlighted. Through the use of Hierarchical Process Modelling (HPM), two key approaches to mitigate jamming were identified: (1) Fault Diagnostics (FD) and (2) Fault tolerant design. The development of a fault tolerant EMA system is currently at an early stage for implementation within safety critical systems due to the increased complexity of such systems (for example the anti-jamming system may require FD functionality itself). Challenges also exist for FD approaches particularly in achieving a robust means of fault detection. It is proposed that a hybrid FD approach, using a combination of model based and data-driven techniques to predict the onset of jamming, would be beneficial in capturing the discrepancies between the predicted and observed behaviour used to isolate and identify faults. Furthermore, several aspects of modelling and of data-driven methodologies for FD in the literature omit potentially important behaviours, and recommendations are made to improve upon this. For example, the simulation of faults in test stand analysis and the fidelity modelling of the motor and mechanical components are key areas to develop.
\end{abstract}

Keywords-Prognostics; Health Monitoring; Aerospace; Ballscrew; Electromechanical Actuators; Jamming

\footnotetext{
Yameen M. Hussain et al. This is an open-access article distributed under the terms of the Creative Commons Attribution 3.0 United States License, which permits unrestricted use, distribution, and reproduction in any medium, provided the original author and source are credited.
}

\section{INTRODUCTION}

EMAs are being increasingly considered by aircraft manufacturers to replace traditional hydromechanical systems. The often-cited drivers for this shift include reduced system weight, ease of maintenance and potential for greater precision in control (Hoffman, Hansen, Beach, Plencner, Dengler, Jefferies \& Frye, 1985). The introduction of EMAs to replace hydraulic systems could make for easier power distribution, using electrical cables in place of hydraulic pipes, as well as eliminating the maintenance infrastructure required for a hydraulic based system (Stridsberg, 2005).

These positive drivers are equally valid for safety critical applications such as primary flight control systems and landing gear systems; however, the absence of reliable failsafe mechanisms and redundancy to mitigate the single point of failure (ballscrew jamming) has made it challenging to introduce EMAs to such systems (Balaban, Bansal, Stoelting, Saxena, Goebel \& Curran, 2009). In this paper the progress made so far in trying to mitigate the onset of EMA ballscrew jamming is evaluated.

\subsection{Background}

Up until the 1970s, electrical power on commercial aircraft was predominantly used on electronic and utility functions with sparse application for other functions (Jones, 1999). Given the advances in permanent magnet materials and power electrical devices, the use of electrically powered applications in place of traditional hydraulics and pneumatics appeared to be more advantageous thus prompting a drive towards the concept of All Electric Aircraft (AEA) near the end of the 1970s (Jones, 1999). 
Studies conducted by NASA in the mid-1980s (Hoffman, et al. 1985) concluded that whilst application of AEA technology is feasible and the benefits of achieving a reduction in operational costs due to the weight saving advantages and maintenance is possible, such wholesale changes would bring about more risk for the conservative and safety driven aerospace industry. This has prompted the industry to opt for an incremental adoption of electrical technology within secondary aircraft systems thus the process is now known as More Electric Aircraft (MEA) (Jones, 1999).

As mentioned, much of the research for MEA has considered replacing actuation systems from traditional hydromechanical actuators to EMAs. Actuators on a typical commercial aircraft are principally found on the flight control surfaces as shown in Figure 1.

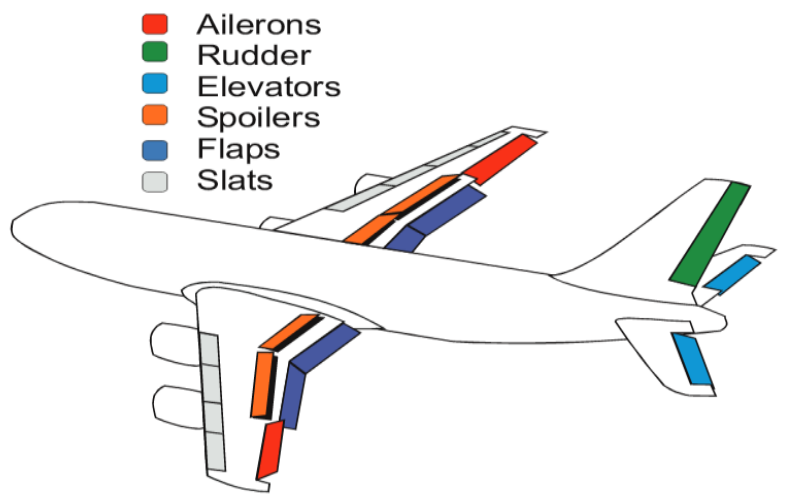

Figure 1. Flight Control Surfaces (Bennett J, 2010).

The ailerons, rudder and elevators are classified as primary control surfaces and are safety critical applications. Another safety critical application that uses actuation systems is the landing gear system, in particular the extension/retraction mechanisms. Figure 2 shows an example of a typical main landing gear system.

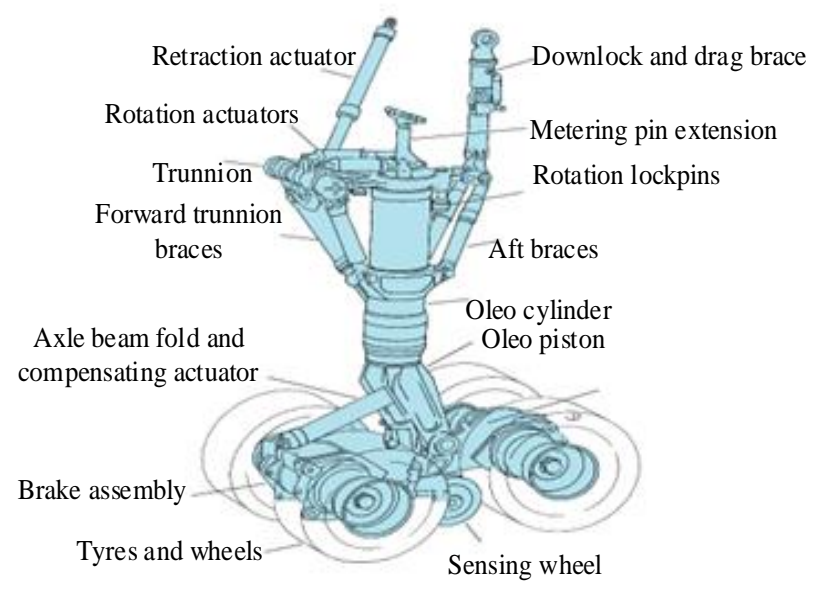

Figure 2. Example Main Landing Gear (Landing Gear parts, 2015).
EMAs consist of a motor, gearing and a ballscrew to provide incremental linear motion powered by the motor. Figure 3 shows a schematic of a typical EMA system.

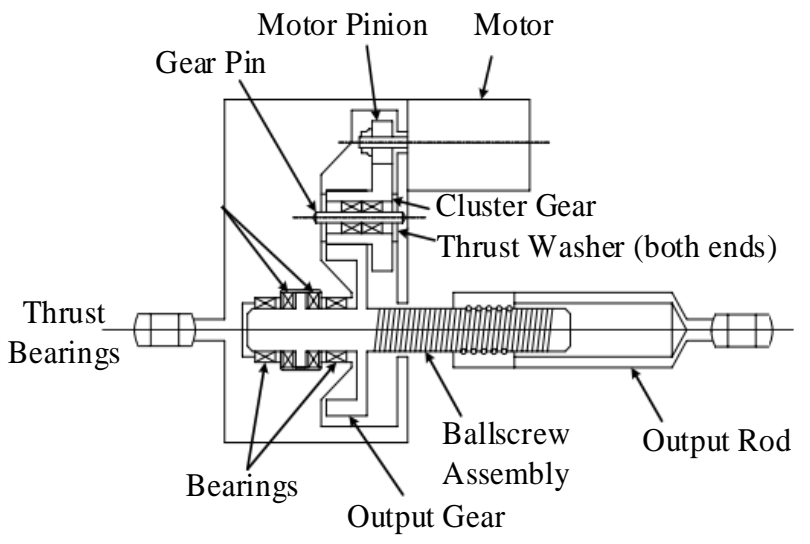

Figure 3. EMA System (Bodden, Clements, Schley \& Jenney, 2007).

EMA ballscrew jamming (a single point of failure) has been identified as a major factor in preventing EMAs from being more thoroughly considered as an actuator for safety critical applications (AIR5713, 2008). Another issue also arises on whether EMA redundancy can be designed to equal the flight safety reliability of dual/triple redundant hydromechanical systems (Leonard, 1984). For example, for an Airbus A320 aircraft landing gear extension/retraction system, loss of all hydraulic systems would result in using gravity extension to mitigate a catastrophic event (Airbus, 1998).

Significant research was conducted through flight test and development programmes in the early 1980s in order to gain more confidence in implementing EMA technology for aircraft actuation systems (Cooper, 2014).

Lockheed and Sundstrand collaborated in a research programme to develop a flight-worthy EMA for an aileron on the Lockheed C-141 military aircraft (Norton, 1986). The EMA replaced a traditional hydraulic actuation system (for starboard aileron) with 14 hours of flight tests conducted in 1986. The flight tests demonstrated feasibility for EMA implementation to primary flight control systems, however, issues were reported relating to variable performance due to temperature and increased sensitivity to autopilot inputs (Norton, 1986).

Lucas Aerospace have also been involved in EMA research development from 1968 with early focus on missile control surfaces (Croke \& Herrenschmidt, 1994). Lucas Aerospace went on to design and develop EMAs for aircraft actuation systems in 1988. The design considered an EMA with a brushless DC motor powered by a 270 VDC bus. The initial design was only implemented for test bench purposes, however, advancements were made with preliminary designs 
factoring in installation to an aircraft envelope (with the assistance of a commuter jet manufacturer) (Cooper, 2014).

Boeing introduced EMAs to aircraft actuation systems to their Boeing 777 aircraft in the early 1990s. The EMAs were implemented as an electrical backup arrangement for the flaps and slats (Rea, 1993). EMAs also feature on the Airbus A380 slats and tail horizontal stabiliser (Adams, 2001). On more recent aircraft, EMAs have also been implemented on the Boeing 787 on 4 (out of 14) spoilers as well as for wheel braking (Mare, 2016). These applications are considered secondary (non-safety critical) aircraft systems.

Given the sparse implementation of EMAs in today's commercial aircraft actuation systems, Electro-hydraulic Actuators (EHA) are considered the intermediate solution between hydromechanical and electromechanical actuation systems (Bennett, 2010). EHAs are essentially a hybrid electrical and hydraulic device where the actuator is hydraulically operated with the hydraulic fluid self-contained and pressurised by an inbuilt motor to drive the actuation mechanism (Churn, Maxwell, Schofield, Howe and Powell, 1998). EHAs are viewed as advantageous over conventional hydraulic systems with increased fluid pressure and power density during actuations (Moir \& Seabridge, 2008). Loss of operation would inhibit the hydraulic rod in exerting a force thus defaulting to damping action allowing for an adequate fail-safe mechanism by enabling other actuators to fulfil the actuation (Bennett, 2010). This makes EHAs the preferred choice in safety critical applications today.

The aims of this paper are to:

1. Provide an evaluation of the current state-of-the-art with regard to mitigating EMA ballscrew jamming.
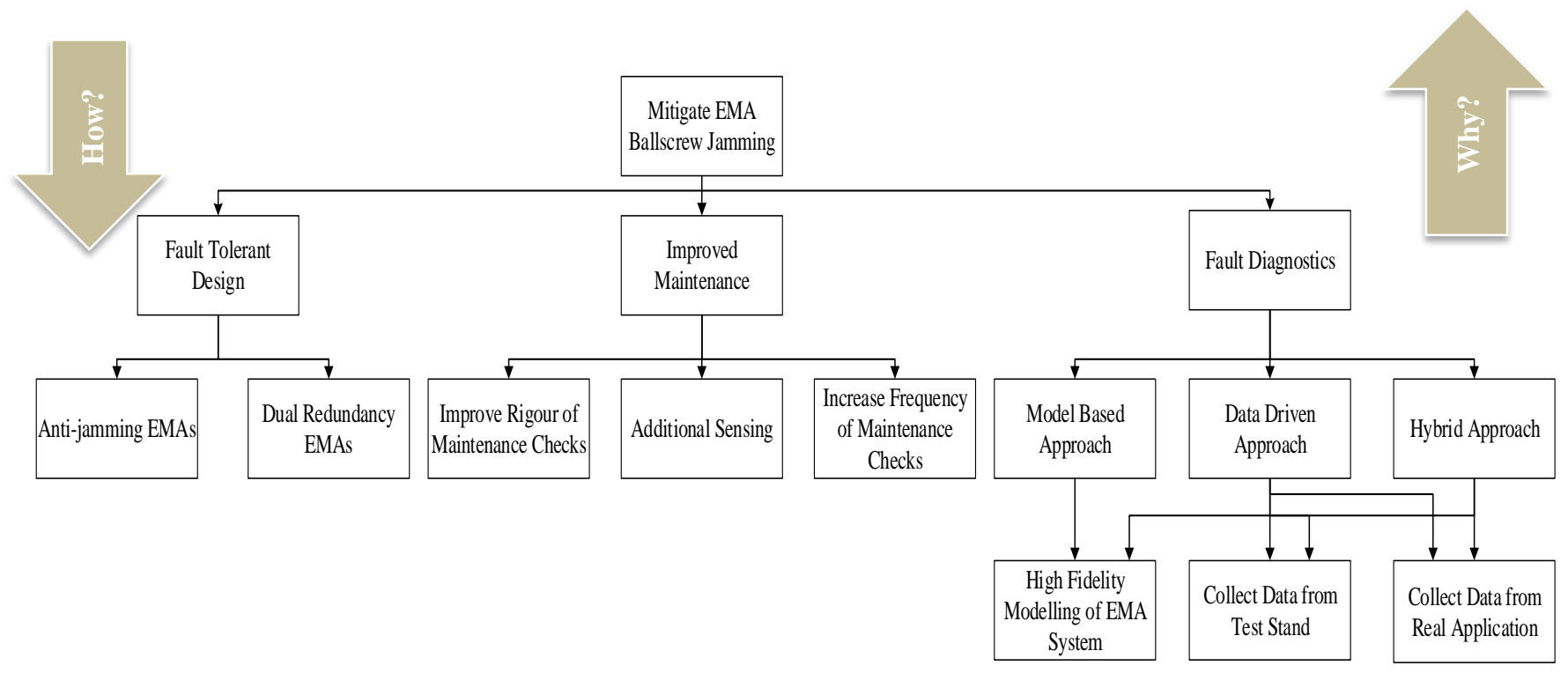

2. Highlight the future challenges to mitigate the onset of EMA ballscrew jamming.

\section{Methods To Mitigate EMA BallsCREW JAMMing}

A Hierarchical Process Model (HPM) was constructed to act as a framework from which to consider existing approaches to mitigate EMA ballscrew jamming.

A HPM can be a useful way to manage complexity to a single problem. A HPM intends to show hierarchy with each level representing a more detailed decomposition of processes indicating transformational entities. HPM is driven by the need to support effective decision making whilst acknowledging issues related to risk and uncertainty. Pidd (2004) identified the need behind HPM by describing nature as being hierarchically organised with emergent properties at various levels of complexities.

The structure of a typical HPM stems from an initial purpose statement, which then branches downwards by exploring how it could be achieved through various system levels, as more detail is added (Checkland \& Poulter, 2007). Figure 4 shows the HPM with purpose statement 'Mitigate EMA ballscrew jamming'. At the same time, it is possible to establish purpose and reasoning of a solution when viewing the HPM 'bottom up' (Checkland \& Poulter, 2007).

The HPM in Figure 4, describe three top-level approaches to mitigate jamming: fault tolerant design, Improved maintenance, and FD. Fault tolerant design and FD are further considered in sections 2.1 and 2.2 of this paper respectively. The 'improved maintenance' node is not considered further in this paper as the methods of analysis have limited similarity with the other two, requiring evaluation of assumed usage profiles and the impact on existing maintenance policy, for example covered by the

Figure 4. Hierarchical Process Model. 
Maintenance Review Board (Robelin, 2010). Additional maintenance actions also incur ongoing costs due to increased labour and aircraft downtime (Jennions, 2012).

\subsection{Fault Tolerant Design}

Fault tolerant design describes a range of techniques, applied at either a system or component level, that provide 'fail operational' or 'fail safe' behaviour, typically through introducing redundancy.

The are several examples in the literature achieving fault tolerant behaviour on electric drive systems (the first section of an EMA) for aerospace applications. For example, at the electrical machine level, this could be achieved with dual/triple motor redundancy or by the use of poly-phase machines. Bennett, Mecrow, Atkinson and Atkinson (2011) suggest that with each lane including an independent converter, a fault tolerant electrical drive can withstand failures associated with power supply and control interface and therefore increasing the number of lanes would skew the EMA reliability figure towards the mechanical components. Figure 5 shows an EMA fault tree with dual lane fault tolerant electric drive derived by Bennett et al. (2011).

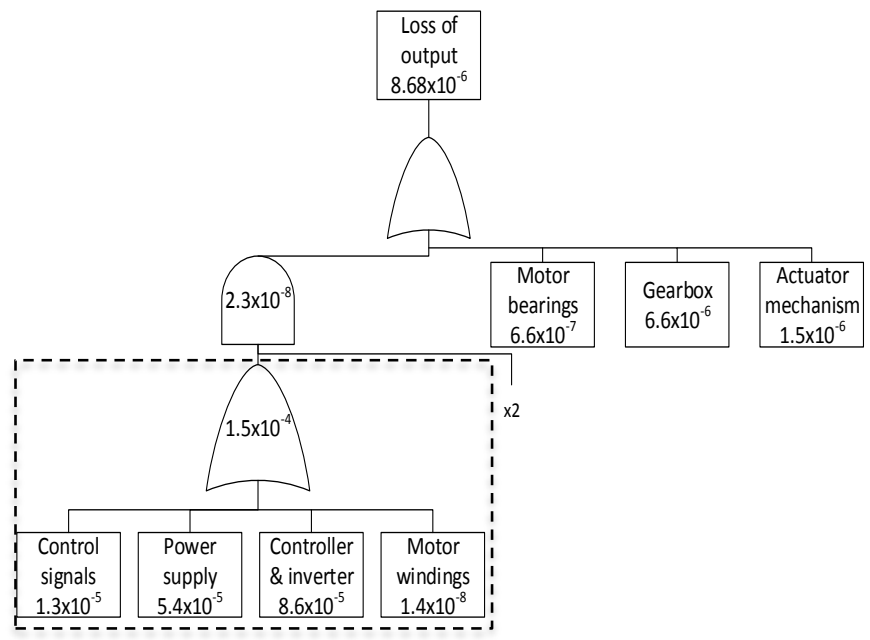

Figure 5. EMA Fault Tree with Dual Lane Fault Tolerant Electric Drive (Bennett et al. 2011).

Providing fail operational behaviour for the mechanical components of the EMA is less well developed and summarised in the following sections.

\subsubsection{Anti-Jamming EMAs}

There have been a few EMA designs which have factored in mechanical modifications in an attempt to prevent jamming.

Cronin (1985) proposed an EMA system with hydraulic coupling as a means to protect against mechanical jamming. The proposed arrangement was such that included an EMA connected to a control surface through an EHA (without a pump).
The backup EHA exerted the same amount of force as the primary EMA, however, such an arrangement adds significant weight and complexity to the overall system thus deeming such solution unsuitable for implementation to safety critical aircraft actuation systems.

(ii) More recently, Nguyen, Behar and Mckay (2014) proposed an EMA design for jam tolerance which incorporates a damper assembly that becomes coupled to the output rod (connected to the moveable surface) during the event of a mechanical jam. This in turn decouples the ballnut from the output rod. The damper system ultimately enables a passive, controlled rate return of the EMA output to a fail-safe position along with a latch that holds the position (within fail-safe mode). The overall process relies on a complex mechanical arrangement which may in turn require additional scheduled maintenance actions and possibly condition monitoring.

\subsubsection{Dual Redundancy EMAs}

There have also been EMA design considerations with builtin redundancy in the event of ballscrew jamming.

Collins and Sunstrand (2004) proposed a dual actuator system acting on a single flight control surface over a summing lever. The summing lever position corresponds with the sum of the positions of the actuators attached to it. Jamming of one of the actuators would result in the other actuator to compensate for the malfunctioning one in order to bring the flight control surface to a neutral position. The proposed design would include two EMAs and a link arm which not only adds weight but increases design complexity.

There is current research by Triumph Actuation Systems U.K Limited, in collaboration with Kugel Motion Limited and Nema Limited, looking into High Availability Redundant Actuation Systems (HARAS) since November 2015 (Triumph Actuation Systems - U.K, Ltd., 2015). The research programme is specific to developing fault tolerant electrical actuation solutions for primary flight control systems on Unmanned Vehicle Aircraft (UAV). This initiative indicates that there is still an industry need to implement EMAs to for flight safety-critical systems.

Aside from achieving a jam-tolerant EMA system, aircraft manufacturers face other technical challenges for EMA implementation to flight safety critical systems. Todeschi (2011) highlights constraints in installation of EMAs (for flight control systems) whereby space may be limited to 
accommodate a complex EMA system architecture. Todeschi (2011) also emphasised that 'weight' would be another constraint during the design phase as well as design complexity which could impact maintenance scheduling and introduce further health monitoring. Given the criteria described by Todeschi (2011), the designs presented for antijamming EMAs could bring about operator concerns on reliability, weight and implementation.

\subsection{Fault Diagnostics}

Fault Diagnostics (FD) is an element in the broader topic of Prognostics and Health Monitoring (PHM), involving the process of identifying an instance of a component or system behavior that is different from the expected behaviour and locating the origin and cause of that behaviour. PHM takes this further by attempting to provide insight into a component's health, and determine its Remaining Useful Life (RUL); PHM can thus increase availability by reducing unscheduled removals and reducing downtime, ultimately reducing Direct Maintenance Costs (DMC) (Jennions, 2012).

There have been several research projects exploring FD to detect the onset of ballscrew jamming both within academia and industry. The following sections evaluate the progress made in FD (for mitigating EMA jamming) considering the main approaches to developing the features correlated to damage i.e. modelling approaches, data-driven (experimental) approaches and hybrids of both.

\subsubsection{Model Based Approaches}

Modelling of a system to understand the physics of failure by monitoring system parameters is viewed as a cheaper alternative and is less time consuming and labour intensive compared to building a corresponding test stand. Figure 6 provides an overview of the processes involved a model based approach in the context of diagnosing system health.

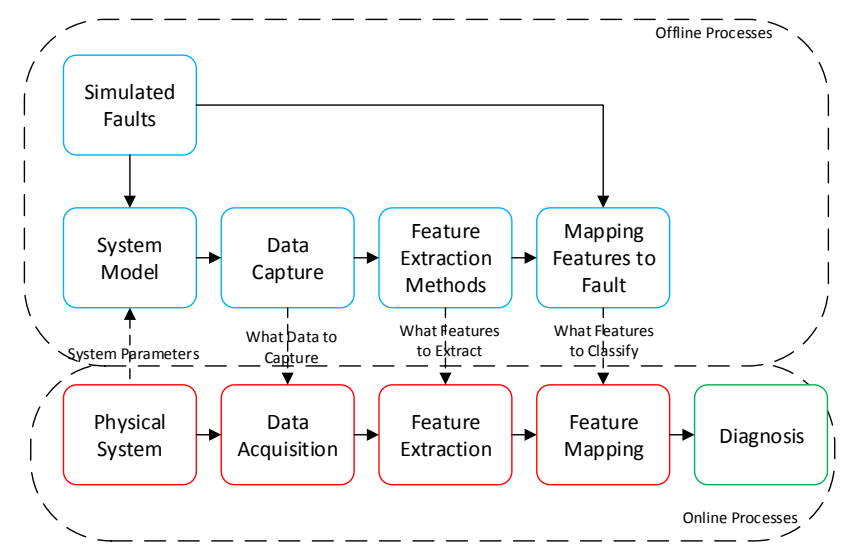

Figure 6. Model Based Approach.

A model-based approach through high fidelity modelling of an EMA system for fault detection and failure prediction has been considered to be a useful preliminary step in understanding system behaviour under normal and abnormal conditions. Modelling an EMA system in detail can enable the prognostics design engineer to trace back failure modes to relatable physical system parameters thus providing the engineer with helpful diagnostic information.

Byington and Stoelting (2004) presented a modelbased approach to PHM for EMAs on flight control actuators. The methodology was centred around diagnosing failures associated to the motor, gear slippage and bearings. Failures were selected based upon the highest number of occurrence from inservice events. A mathematical dynamical model of the EMA system was developed using Matlab/Simulink of which was linked to the physical processes that drive the health monitoring of the EMA. This included emphasis on modelling friction co-efficients at key elements of the EMA drivetrain such as the motor, gearbox and ballscrew. This was varied to understand the impact on response time, motor current and load.

(ii) Maggiore, Vedova, Pace and Desando (2014) developed a Matlab/Simulink model of an EMA system to be utilised for fault analysis associated with mechanical failures due to progressive wear; this includes friction, backlash, coil short circuit and rotor static eccentricity. The research was focused on characterising and building systemrepresentative models for these failure modes. The modelled EMA system was typical of an arrangement for a primary flight control system comprising a control and power drive electronics, a Brushless Direct Current (BLDC) motor, gearing and a ball/rollerscrew. Motor current, angular speed and position were the parameters being monitored. Subsequent failure maps were derived for fault detection/evaluation based on simulations of the different types of failures.

A high fidelity and exhaustive model of the system features can enable identification of parameters that are associated to the build-up of a specific failure mode. This can therefore allow utilization of parametric estimation for diagnostics application and state of health estimation, however, this is dependent on the level of modelling effort and granularity.

Whilst modelling is a useful means to get an initial perspective of a system, it is never a 'true' representation of the actual behaviour. For instance, Byington and Stoelting's (2004) approach utilized variation of friction coefficients as a means to perform sensitivity analysis to evaluate mechanical losses in the drivetrain. The reality, however, is that friction is prevalent in many areas of the drivetrain, therefore, it would be difficult to ascertain the location of the friction build-up. It would require one to quantify the amount of mechanical losses attributed due to friction by mitigating the effects of external loads, backlash and any other 
unwanted non-linear effects. It is therefore imperative that in the case for modelling the physics of failure behind ballscrew jamming, a more robust approach is taken in terms of modelling wear and friction by considering the most contentious areas of friction within such systems.

Maggiore et al. (2014) gave importance to the build-up of friction as a pre-cursor to the onset of jamming. The corresponding failure maps of motor current provided useful information in terms of evaluating friction torque at a system level by assigning thresholds for the onset of a failure. It, however, was not clear whether friction monitoring at local levels for contentious contact regions in the ballscrew (ball and nut, and ball and screw) could be characterisable especially when trying to diagnose for jamming faults from these contact areas.

\subsubsection{Data-Driven Approaches}

A data-driven approaches to FD can be split up into two methods:

(a) Retrieving data from a real application such as an inservice EMA;

(b) Retrieving data from a representative test stand.

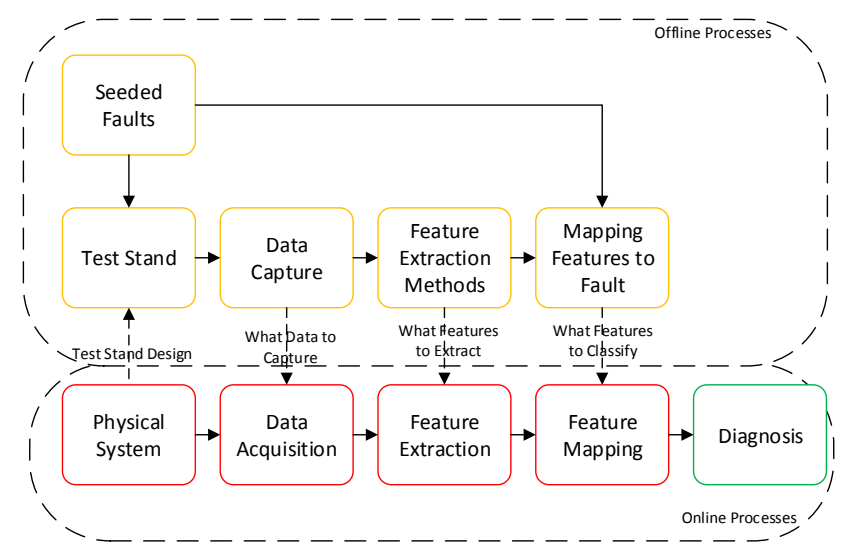

Figure 7. Data-Driven Approach.

Obtaining data from an in-service EMA can be seen as advantageous as the information generated will be a true representation of the application usage profile and system behaviour. By this, aerodynamic loads and other environmental effects are factored in. The issue, however, is the limited nature in which the data is obtained. Aircraft manufacturers and operators are reluctant to have additional sensing due to added weight implications and reliability (Donald, Garg, Hunter, Guo \& Semega, 2004), thus requiring diagnostics engineers to isolate a problem like jamming within the EMA drivetrain based on existing sensor signals alone. Such approach, however, is considered within a PHM framework through a combination of physical modelling and test stand data, of which is discussed later in this paper.
The building of a bespoke EMA test stand can enable run-tofailure tests as well as seeded failure tests to be performed. The advantage here is that more sensors can be added to improve the understanding of the system behaviour as well as characterise different types of failures modes. However, the data is limited by the ability of a realistic failure to be replicated. A significant amount of research has been conducted in this area with particular focus on seeded failure tests to EMA test stands.

Bodden et al. (2007) seeded contaminant to an EMA test stand and cycled it until failure. The amount of debris was the key parameter for setting the rate at which a jamming would occur. A measure of actuator efficiency was quantified by taking the ratio of power output and power input of the system. It was found that as heat and vibration energy increased, the power input to the system increased and therefore increased the motor current demand. The test stand was also fitted with other sensors in order to identify other pre-cursors such as temperature and vibration in addition to motor current. Temperature readings were recorded (thermistors mounted on the rear of the motor housing) with increased temperatures observed which were attributed to the higher level of friction in the system due to the induced debris. Such readings, however, were not characterisable against the nature of the simulated fault therefore making it difficult to isolate the actual location of the increased friction in reality.

(ii) An EMA test stand was built with airworthy equipment in which in-flight data was post processed on the ground (Balaban, Saxena, Goebel, Byington, Watson, Bharadwaj \& Smith, 2009). This followed the philosophy of taking the data off aircraft and performing prognostics on the ground. Jamming faults were simulated on the test stand with results showing good agreement with developed thermal and mechanical models. The issue, however, was the abrupt nature in which the jamming occurs making it challenging to design a prognostics algorithm based on such data. Using the same test stand, Balaban, Saxena, Narasimhan, Roychoudhury, Goebel and Koopmans (2010) also introduced spalling to the ballscrew to understand the effects on the system response of the EMA. Indentations were created in the test ballscrew at high stress contact points at dimensions of $0.3 \mathrm{~mm}$ depth and widths ranging from $0.3-0.5 \mathrm{~mm}$ to evaluate how the size of the initial spall affects the nature of its growth. An accelerometer was fitted to the nut of the ballscrew to monitor the frequency of the system. The results showed that there was increased vibration in the ballscrew due to the induced spalling. 
Unless certain fault modes can be characterised through EMA test stand analysis, it may be challenging to isolate and identify a particular fault mode such as ballscrew jamming.

Additionally, the failure analysis conducted through experimental analysis has largely involved simulating faults. By this, faults were artificially implemented through seeding debris and introducing structural damage to the ballscrew. Run to failure tests are an effective means to obtain data corresponding to naturally occurring faults. However, this can be time consuming and expensive, particularly when trying to obtain data for ballscrew jamming, as other failure modes may manifest in performing run to failure tests.

\subsubsection{Hybrid Approach}

The methods discussed so far have solely considered a modelling approach or a data-driven approach in isolation. Narasimhan, Roychoudhury, Balaban and Saxena (2010) explained that a modelling approach works well for deriving analytical models for specific faults only. The data-driven approach requires a lot of data under varying experimental conditions for training a classifier. Furthermore, the classifier would need to consider all types of faults and other conditions, therefore increasing the size and complexity of the classifier.

A hybrid approach to fault diagnostics would entail employing a detailed model of an EMA system against an equivalent physical EMA system to capture any discrepancies from normal behaviour. This would then enable one to identify and isolate faults to prompt further investigation.

Narasimhan et al. (2010) presented a hybrid diagnostic approach that involved the fusion of model-based and datadriven based methods. The data-driven method was based on the previously built flyable EMA test stand by Balaban et al. (2009). A top-level diagram of the hybrid approach followed is shown in Figure 8.

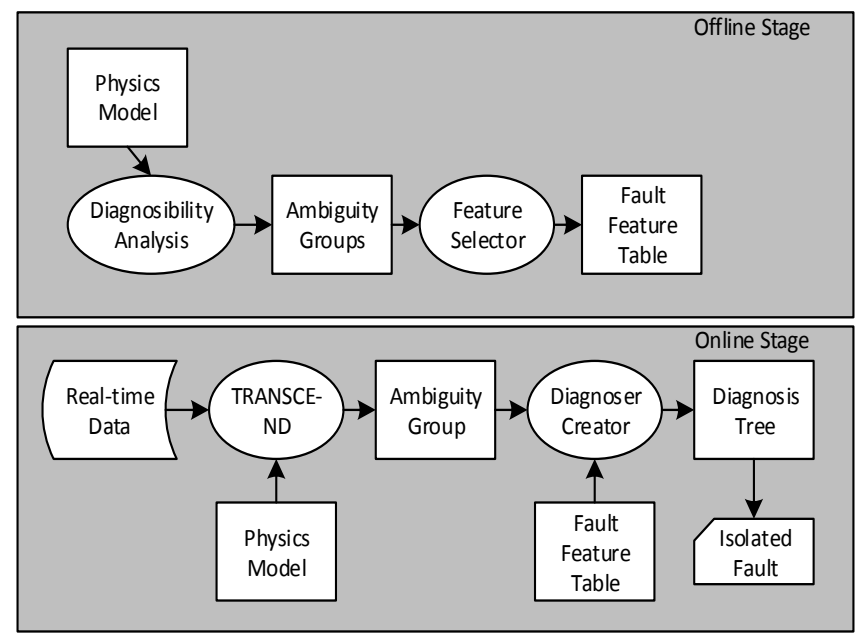

Figure 8. Hybrid Diagnostics (Narasimhan et al. 2010).
The hybrid diagnostics approach presented by Narasimhan et al. (2010) not only combined model-based and data-driven methods, but considered offline and online stages in the diagnostic process. The offline stage involved use of an EMA system physical model to generate repeatable fault signatures, which were then categorised into fault feature tables. The online stage utilised real-time data (from the flyable EMA test stand) from which anomalies were detected and isolated using the physical model before classifying ambiguity groups.

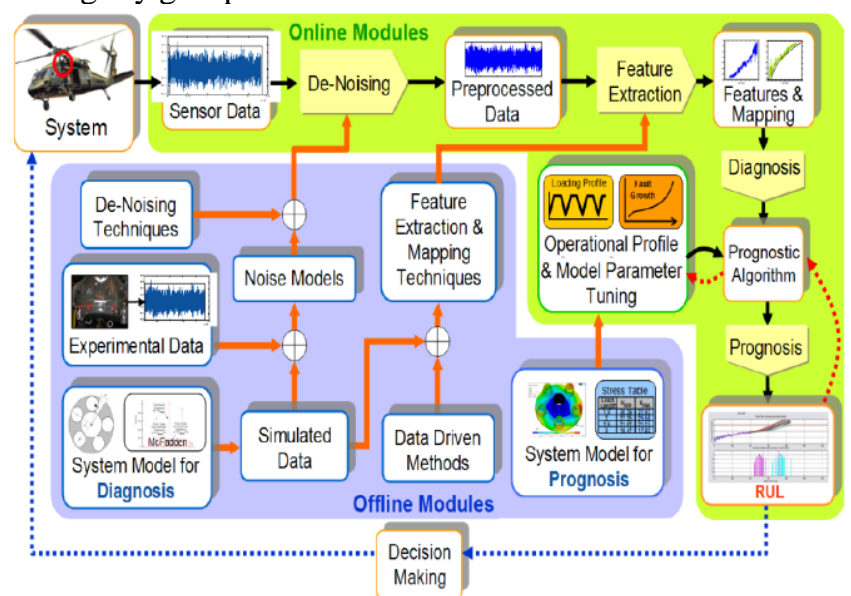

Figure 9. Data Analysis and Decision Making (Saxena, 2010).

Figure 9 provides a holistic view of the processes involved in PHM as presented by Saxena (2010). The online modules involve a sequence of data acquisition and manipulation, state detection, health assessment, prognosis assessment and decision making. Emphasis is given to what can be learned from the offline modules.

The offline modules are dependent on a fusion of data from experimental analysis and system modelling. A data fusion approach can be seen as advantageous as the combination of modelling and data-driven analysis can help to isolate and identify certain types of faults by comparing predicted and observed system behaviour.

There are limitations in obtaining in-service EMA data due to manufacturers' concerns in reliability and adding more sensors (due to space and weight constraints) (Donald et al, 2004). Therefore, in the context of mitigating EMA ballscrew jamming, a combination of a high fidelity EMA model and extraction of relevant test stand data is proposed as the optimal approach in performing fault diagnostics for this case. Figure 10 provides an overview of the proposed hybrid approach for EMA ballscrew fault diagnostics. Critically here the parallel test stand and simulated system models provide complimentary functions: the test stand providing true behaviours for a limited number of conditions that can be used to tune the system model, which in turn can provide simulations of broad ranging operational conditions. 


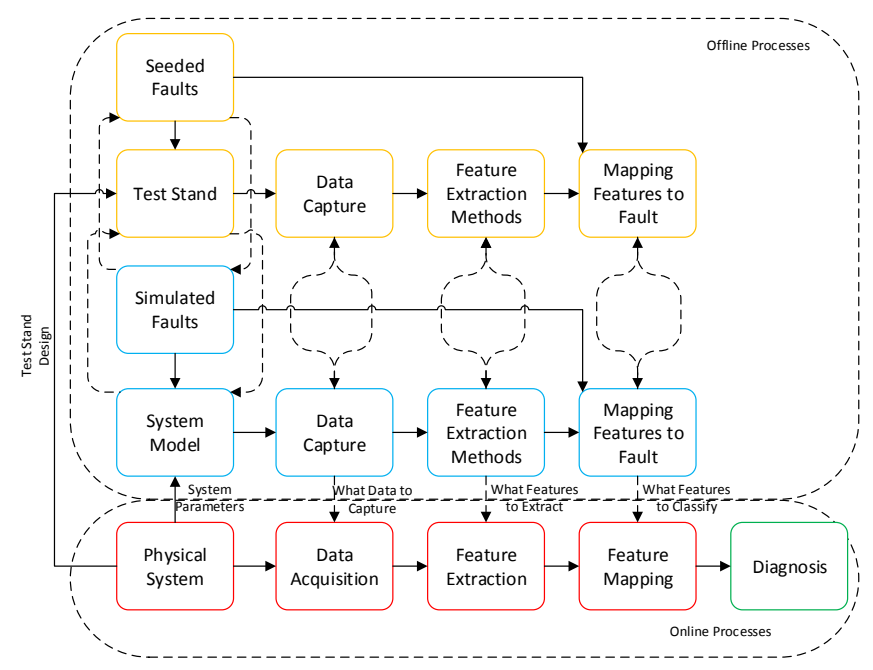

Figure 10. Hybrid Approach to EMA Fault Diagnostics.

\section{Challenges To Be Addressed}

Out of the strategies evaluated, many challenges remain in trying to mitigate the onset of EMA ballscrew jamming. Fault Tolerant EMA designs are considered to be at an early stage with work to develop a redundant system for aircraft safety critical systems still ongoing.

The combination of a model based and data-driven approach can improve the overall prediction accuracy, therefore, a hybrid approach to fault diagnostics of the EMA jamming case was considered to be the optimal approach. The success of the hybrid approach would, however, still rely on the robustness of the modelling and data generated from an EMA test stand and/or real application. This section highlights challenges which need to be addressed in trying to create a more robust fault diagnostics algorithm.

\subsection{Challenges for Data-Driven Approaches}

Most reported examples have used seeded fault tests to EMA ballscrews by introducing debris as well as physical damage to the screw. Technical challenges were identified in trying to characterize fault modes from seeded faults as well as reproducing more realistic fault cases.

\subsubsection{Ballscrew Thermal Expansion}

Previous research has investigated the effects of seeding debris and damage to the ballscrew to simulate wear. Consideration should also be given to simulate the effects of ballscrew ball deformation due to thermal expansion. The balls within the ballnut of a ballscrew system can undergo thermal expansion due to heat caused by friction (Jeong \& Park, 1992). This can lead to a degradation in performance and positioning accuracy. Figure 11 shows an example of temperature variations due to different ballnut preloads and
Thermal Contact Conductance (TCC) using finite difference methods.

Such conditions could be considered in test stand analysis by seeding deformed or appropriately larger balls in the ballscrew to simulate and evaluate the effects of ball deformation due to thermal expansion.

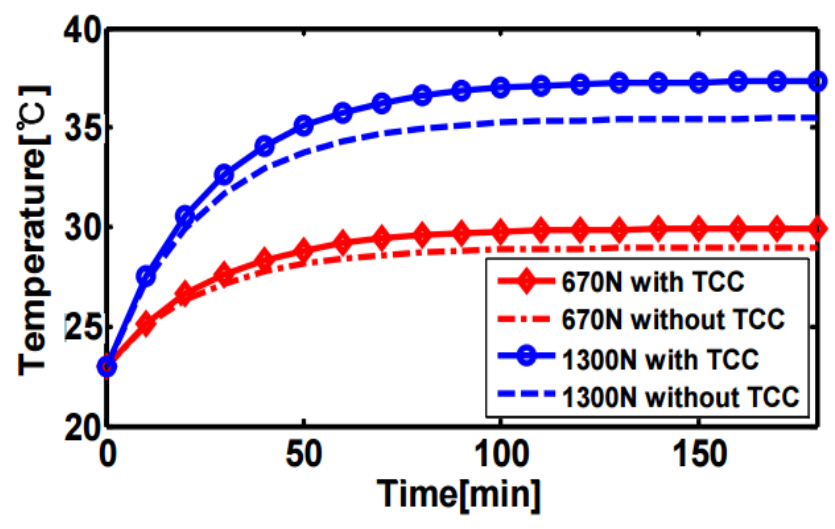

Figure 11. Temperature Variations due to Varying Nut Preloads and TCC (Min, Park \& Chung 2016).

\subsubsection{Seeded Failure Tests}

Whilst useful information can be obtained from simulating seeded faults to a healthy actuator (Balaban et al, 2009) limitations still exist in understanding the true nature from which a particular failure mode may initially manifest. Therefore, more run to failure tests could be conducted to learn from naturally occurring faults and to validate seeded fault test results. This should include the re-use of older actuators that would have started to exhibit wear and degradation naturally from in-service application. This could enable one to distinguish and characterise properties for systems with lower mechanical efficiencies as well as to validate seeded fault test results.

\subsection{Challenges for Model Based Approaches}

In the reviews of previous research, a clear theme emerged that a high-fidelity approach is required for model based approaches to identify the onset of jamming.

\subsubsection{Gearbox Modelling}

For direct drive EMA systems, the absence of gearing simplifies the analysis in diagnosing ballscrew related failures (Gerada \& Bradley, 2008). For gear driven EMA systems, previous modelling approaches have modelled the gearbox using efficiency terms to model losses. Losses in the gearbox would need to be modelled in more detail to account for non-linearities. Losses in gear systems are primarily attributed to gear tooth friction and lubrication churning losses (Schlegel, Hösl , \& Diel, 2009). Detailed modelling of 
these losses would improve the accuracy in decomposing losses across the drivetrain.

\subsubsection{Motor Modelling}

EMA systems have mostly been modelled using simple motor models, with only a few exceptions when more complex models have been used. The investigation with simple models are limited to analysing electromagnetic torque and motor current as being directly proportional without considering motor dynamic behaviour. The research conducted by Maggiore et al. (2014) provides an advancement to this by modelling a BLDC system with power drive electronics that evaluated the torque generated by the motor as a function of the voltages generated by a 3phase electrical power regulator. For future model based approaches, it is proposed that the modelling could consider Field Oriented Control (FOC) techniques, which can capture magnetic behaviour. FOC can be applied where 3-phase AC quantities $\left(\mathrm{I}_{\mathrm{A}}, \mathrm{I}_{\mathrm{B}}, \mathrm{I}_{\mathrm{C}}\right.$ ) can be reduced to DC quantities $\left(\mathrm{I}_{\mathrm{D}}, \mathrm{I}_{\mathrm{Q}}\right)$ using Park's transform (Park, 1929).

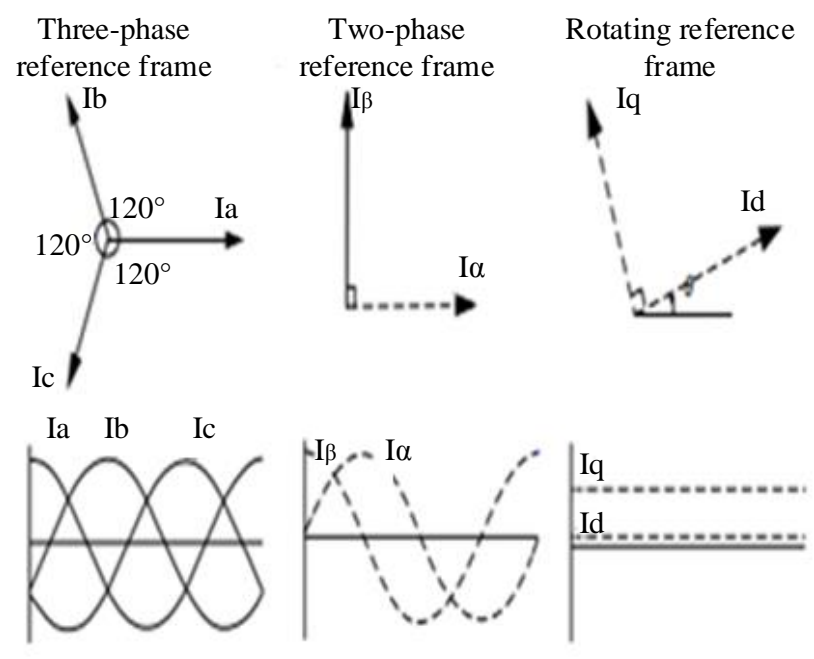

Figure 12. Reference Frames.

This can enable simplified analysis of the DC quantities which can provide in-depth understanding of motor dynamics (such as inductance saliency) for condition monitoring and fault detection within the EMA drivetrain.

\subsubsection{Ballscrew Kinematics}

Buildup of friction is considered a pre-cursor to EMA ballscrew jamming (Balaban, et al. 2009). Model based approaches have often parametrized friction through industry standard coefficients. It is therefore proposed that modelling the most contentious areas of friction within the ballscrew (ball and nut, and ball and screw (Vahid-Araghi \& Golnaraghi, 2011)) to a high fidelity could improve the characterisation of such features for fault detection. This could be achieved by modelling the ballscrew kinematics in more detail by considering the ball and nut, and the ball and screw interactions as shown in Figure 13.

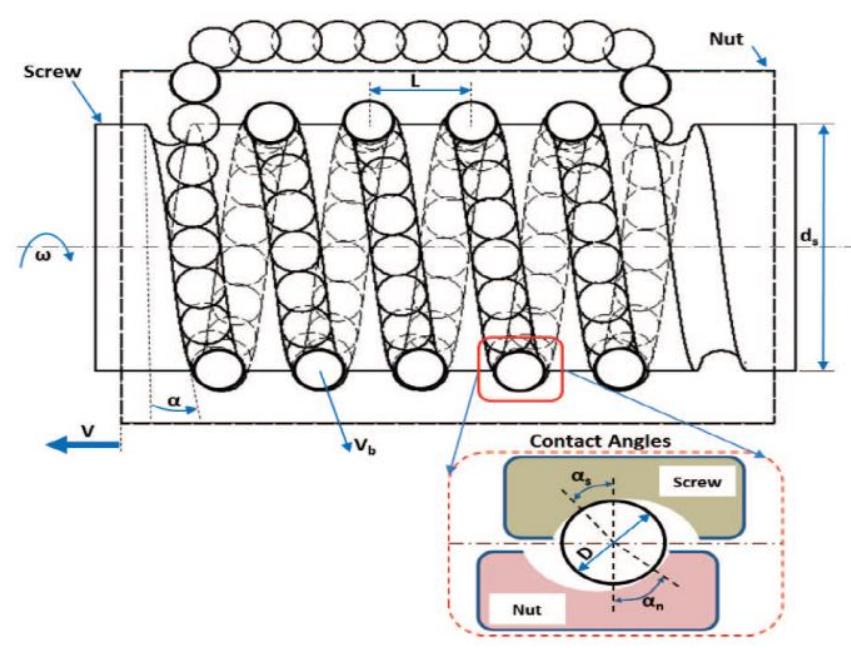

Figure 13. Ballscrew Kinematics (Ismail, Balaban, \& Spangenberg, 2016).

This can enable a more accurate representation of the contact mechanics between the ball and nut, and ball and screw and also consider the effects of slip.

\section{Conclusions}

Various approaches to mitigate EMA ballscrew jamming have been evaluated in this paper. Through HPM, achieving a fault tolerant EMA system as well as FD were considered the two key ways to prevent the jamming case.

A review of literature showed that implementation of a fault tolerant EMA design to aircraft safety critical systems was still at an early development stage due to aircraft manufacturer concerns to do with weight, reliability and installation constraints.

Literature and past research on fault diagnostics methodologies (mainly modelling and data-driven approaches) were also evaluated. It was viewed that a hybrid approach to diagnosing EMA ballscrew jamming faults could be most optimal. The performance of this approach could be maximized through data fusion between a model and experimental data in order to capture discrepancies between predicted and observed behaviour to then isolate and identify the fault from which would prompt further investigation. This is dependent on the granularity of the model as well as the observability of the test stand data.

In order to improve the robustness of the hybrid approach, five recommendations were made in this paper. This included modelling the EMA to a high fidelity with emphasis on modelling the most contentious areas of friction in order to improve characterisation of impending jamming faults. It was also proposed to consider learning from naturally occurring faults (as opposed to simulating seeded faults) by re-using 
older actuators that have started to exhibit wear from inservice application.

\section{ACKNOWLEDGEMent}

This work was supported by the Systems Centre and the EPSRC funded Industrial Doctorate Centre in Systems (Grant EP/G037353/1) and Stirling Dynamics.

\section{REFERENCES}

Adams, C. (2001, October 1). A380 'More Electric Aircraft'. Avionics Magazine.

AIR5713. (2008). In-service Reliability Data of Continuously Active Ballscrew and Geared Flight Control Actuation Systems. SAE.

Airbus. (1998). A319/A320/A321 Flight deck and systems briefing for pilots. Airbus.

Balaban, E., Bansal, P., Stoelting, P., Saxena, A., Goebel, K., \& Curran, S. (2009). A Diagnostic Approach for Electro-Mechanical Actuators in Aerospace Systems. IEEE Aerospace.

Balaban, E., Saxena, A., Goebel, K., Byington, C., Watson, M., Bharadwaj, S., \& Smith, M. (2009). Experimental Data Collection and Modelling for Nominal and Fault Conditions on Electromechanical Actuators. IJPHM.

Balaban, E., Saxena, A., Narasimhan, S., Roychoudhury, I., Goebel, K., \& Koopmans, M. (2010). Airborne Electro-Mechanical Actuator Test Stand for Development of Prognostic Health Management Systems. Annual Conference of the Prognostics and Health Management Society 2010. PHM.

Bennett, J., (2010). Fault Tolerant Electromechanical Actuators for Aircraft. Doctoral Dissertation, Newcastle University, Newcastle, United Kingdom. Retrieved from https://theses.ncl.ac.uk/dspace/handle/10443/990

Bennett, J., Mecrow, B., Atkinson, D., \& Atkinson, G. (2011). Safety-critical Design of Electromechanical Actuation Systems in Commercial Aircraft. IET Electric Power Applications, 37-47.

Bodden, D., Clements, S., Schley, B., \& Jenney, G. (2007). Seeded Failure Testing and Analysis of an Electromechanical Actuator. Aerospace Conference (pp. 1-8). IEEE.

Byington, C., \& Stoelting, P. (2004). A Model-Based Approach to Prognostics and Health Management for Flight Control Actuators. 2004 IEEE Aerospace Conference (pp. 3551-3562). IEEE.

Checkland, P., \& Poulter, J. (2007). Learning for Action: A Short Definitive Account of Soft Systems Methodology, and itsd use for Practitioners, Teachers and Students. John Wiley.

Churn, P., Maxwell, C., Schofield, N., Howe, D., \& Powell, D. (1998). Electro-hydraulic Actuation of Primary
Flight Control Surfaces. IEE Colloquium on All Electric Aircraft, (pp. 3/1-3/5).

Collins, A., \& Sunstrand, H. (2004). USA Patent No. US 6776376 B2.

Cooper, M. (2014). Simulating Actuator Energy Demands of an Aircraft in Flight. Cranfield University.

Croke, S., \& Herrenschmidt, J. (1994). More Electric Initiative - Power by Wire Actuation Alternatives. IEEE Proceedings of the National Aerospace and Electronics Conference (pp. 1338-1346). IEEE.

Cronin, M. (1985). USA Patent No. US 4530271A.

Department of Defense. (1980). Procedures for Performing a Failure Mode, Effects and Criticality Analysis. Department of Defense, USA.

Donald, S., Garg, S., Hunter, G., Guo, T., \& Semega, K. (2004). Sensor Needs for Control and Health Management of Intelligent Aircraft Engines. NASA.

Gerada, C., \& Bradley, K. (2008). Integrated PM Machine Design for and Aircraft EMA. IEEE Transactions on Industrial Electronics, 3300-3306.

Hoffman, A., Hansen, I., Beach, R., Plencner, R., Dengler, R., Jefferies, K., \& Frye, R. (1985). Advanced Secondary Power System for Transport Aircraft. NASA.

Ismail, A., Balaban, E., \& Spangenberg, H. (2016). Fault Detection and Classification for Flight Control Electromechanical Actuators. Aerospace Conference. IEEE.

Jennions, I. (2012). Integrated Vehicle Health Management: Business Case Theory and Practice. SAE International.

Jeong, S., \& Park, J. (1992). Thermal Expansion Analysis of the Ball Screw System by Finite Difference Methods. Journal of the Korean Society for Precision Engineering, 44-57.

Jones, R. I. (1999). The More Electric Aircraft: The Past and the Future? IEE Colloqium on Electrical Machines and Systems for the More Electric Aircraft, (pp. 1/11/4).

Landing Gear parts. (2015). Retrieved from Pininterest: https://uk.pinterest.com/pin/488359153317845635/

Leonard, J. (1984). All-Electric Fighter Airplane Flight Conrol Issues, Capabilities and Projections. IEEE Transactions on Aerospace and Electronics Systems, 234-242.

Maggiore, P., Vedova, M., Pace, L., \& Desando, A. (2014). Definition of Parametric Methods for Fault Analysis applied to an Electromechanical Servomechanism affected by Multiple Failures. European Conference of the Prognostics and Health Management Society 2014. PHM.

Mare, J. (2016). Aerospace Actuators 1: Needs, Reliability and Hydraulic Power Solutions. Wiley-ISTE.

Min, B., Park, C., \& Chung, S. (2016). Thermal Analysis of Ballscrew Systems by Explicit Finite Difference 
Method. Transactions of the Korean Society of Mechanical Engineers, Volume 40, 41-51.

Moir, I., \& Seabridge, A. (2008). Aircraft Systems: Mechanical, electrical, and avionics subsystems integration. John Wiley and Sons, Ltd.

Narasimhan, S., Roychoudhury, I., Balaban, E., \& Saxena, A. (2010). Combining Model-Based and FeatureDriven Diagnosis Approaches - A Case Study on Electromechanical Actuators. 21st International Workshop on Principles of Diagnosis.

Nguyen, D., Behar, B., \& Mckay, T. (2014). USA Patent No. US 8794084 B2.

Norton, W. (1986). Advanced Electromechanical Actuation System (EMAS), Flight Test. USAF.

Park, R. (1929). Two Reaction Theory of Synchronous Machines. AIEE Transactions 48, 716-730.

Pidd, M. (2004). Systems Modelling: Theory and Practice.

Pillay, P., \& Krishnan, R. (1991). Application Characteristics of Permanent Magnet Synchronous Motors and Brushless DC Motors for Servo Drives. IEEE Transactions on Industry Applications, 986-996.

Rea, J. (1993, August). Boeing 777 High Lift Control System. IEEE AES Systems Magazine, pp. 15-21.

Robelin, O. (2010, January). Maintenance Review Board Process (MRB) and Instructions for Continuied Airworthiness. Retrieved from EASA: https://www.easa.europa.eu/system/files/dfu/ws_pr od-g-doc-Events-2010-jan-19-Ref-9.-MRBprocess.pdf

Saxena, A. (2010). Prognostics, The Science of Prediction. Portland.

Schlegel, C., Hösl , A., \& Diel, S. (2009). Detailed Loss Modelling of Vehicle Gearboxes. Proceedings 7th Modelica Conference. Como: The Modelica Association.

Stridsberg, L. (2005). Low Weight, Highly Reliable AntiJamming Device for Electromechanical Actuators. Texas: SAE Technical Paper Series.

Todeschi, M. (2011). Airbus - EMAs for Flight Controls Actuation System - An Important Step Achieved in 2011. SAE International.

Triumph Actuation Systems - U.K, Ltd. (2015). High Availability Redundant Actuation Systems - HARAS. Retrieved from Gateway to Research: http://gtr.rcuk.ac.uk/projects?ref=102374

Vahid-Araghi, O., \& Golnaraghi, F. (2011). Friction Induced Vibration in Lead Screw Drives. Springer.

\section{BIOGRAPHIES}

Yameen Monsur Hussain received a BEng (Hons) in Aviation Engineering from Brunel University, London in 2010 and MSc in Fluid Power Systems from the University of Bath in 2011. He is currently an Engineering Doctorate (EngD) student at the University of Bristol studying Health Monitoring of Electrical Actuation Systems in collaboration with Stirling Dynamics Ltd. His current research interests are prognostics and health management techniques, reliability, electromechanical actuation, tribology and optimisation of maintenance scheduling.

Stephen G Burrow received the MEng and PhD degrees in electrical engineering from the University of Bristol in 1998 and 2002, respectively. He is currently a Reader in the Department of Aerospace Engineering, University of Bristol. His current research encompasses power electronics, machines and energy harvesting, and environmental sensing.

Leigh Henson received a BEng (Hons) degree in Mechanical Engineering from the University of Huddersfield in 2002 following successful completion of a four year course including a twelve month industrial placement at Agusta Westland Helicopters (now Leonardo). Awarded Chartered Engineer (CEng) status as a member of the Institute of Mechanical Engineers (IMechE) in 2006 after four years post graduate experience in the civil aerospace industry with Airbus. Working for Stirling Dynamics has built extensive experience in Landing Gear Systems covering research and technology, new aircraft/equipment development and qualification including: requirements capture, $V \& V$ process, first flight, flight test, certification, entry into service (EIS) and continuous product development (CPD).

Patrick Keogh received degrees from the universities of Nottingham and Manchester. $\mathrm{He}$ was a Research Technologist at the Engineering Research Centre, GEC Alsthom (now Alstom). In 1990, he joined the Department of Mechanical Engineering, University of Bath, Bath, U.K. His current research interests include rotor dynamics, magnetic bearing systems, active vibration control, modern optimal control for multivariable systems, contact dynamics, and associated thermal behaviour of bearings. 